\title{
An Efficient Traffic Control System and License Plate Detection Using Image Processing
}

\author{
Dr. Govind Shah \\ Professor, Department of Electronics Engineering, A.K.B.M.C Ranjpur India \\ researchergovindshah@gmail.com
}

\begin{tabular}{|c|c|}
\hline Article History & Abstract \\
\hline $\begin{array}{l}\text { Article Submission } \\
19 \text { December } 2016 \\
\text { Revised Submission } \\
1 \text { February } 2017 \\
\text { Article Accepted } \\
10 \text { March } 2017 \\
\text { Article Published } \\
31^{\text {st }} \text { March } 2017\end{array}$ & $\begin{array}{l}\text { Automatic license plate recognition is extracted from license plate of the vehicle. It is } \\
\text { taken as an image or a continuous image taken in sequence. The extracted } \\
\text { information can be with or without a database in many applications like electronic } \\
\text { payment systems and freeway and arterial monitoring devices for traffic surveillance. } \\
\text { ALPR employs CC camera, advanced camera or black and white, color camera to } \\
\text { capture the image. ALPR is fruitful if the captured images are of good quality. ALPR } \\
\text { is a real time application that processes the images of license plates in various } \\
\text { conditions like dark or bright times in a day. A general technique should be identified } \\
\text { to process images in many different countries or states. We should know that the } \\
\text { license plate generally consists of various colors, languages, fonts and others have } \\
\text { images in the background. Also, these plates are obstructed by mud, light, some } \\
\text { accessories especially on a car. Here, we discuss about methods for ALPR. We } \\
\text { classify ALPR based on the features they are used in each method and knowing their } \\
\text { advantages, disadvantages, recognition accuracy and processing speed. Managing } \\
\text { the timing in traffic controlling by calculating the density of an image. } \\
\text { Keywords: License plate, Traffic light control, Background subtraction, Automatic } \\
\text { license plate recognition. }\end{array}$ \\
\hline
\end{tabular}

\section{Introduction}

Here, we are using an embedded system and image processing technique. An embedded system is a system that generates functionality in an electrical system, preferably with real- time computing constraints. It is an embedded device in parts, preferably having mechanical and hardware components. Nowadays, an embedded system takes hold on many systems at a time [1]. Recently, embedded systems consist of a microcontroller. Even though simple microprocessors are also employed in various sophisticated applications. In either case, processors used may be types ranging from general purpose to very specialized in certain class of computations, or even custom designed for the application at hand. Physically, they are starting from mobile devices like smart watches, to large stationary installations like traffic lights, factory controllers and largely complex systems like hybrid vehicles, MRI, and avionics. Complexity varies with one microcontroller to many different units; components are mounted inside a large chassis [2].

An image processing is a form of signal processing in which image is given as an input, the output may be an image or components of an image after processing is done. In image processing, we consider an image as a 2-D signal that is processed by employing various techniques to it. Image processing is nothing but a digital image processing. As per the survey nowadays, many vehicles are being theft. Though the technology has developed still the theft vehicle cannot be identified easily. In order to avoid the disadvantage, we have implemented an innovative method to know the theft vehicle by using an image processing technique. Also, in addition, we are managing the traffic signal time interval by computing the density of vehicles. It is accomplished by using object detection algorithm. The traffic lights can be controlled according by its output [3]. 
Almost all the traffic light systems functions based on the mechanism of time which alters the lights at regular basis. A traffic light system generally identifies the vehicle present or not and do the need accordingly. The basic key point behind this system is that the vehicle drivers may not spend much time unnecessarily for the lights to alter [4].

\section{Related Work}

ANPR is a system that captures the license plate of a vehicle. Stolen automobiles can be identified based on ANPR in an effective way especially on the highway roads. The license plate number is identified by an algorithm known as number plate locale method. Edge Finding Method and Window Filtering Method offers better improvement in the license plate number system identification. The advantages are there is no difficult in feature identification like noise in the image, variation in the luminance. Hsieo-chu, a scientist proposed in which a past number plate identification is an important aspect in applications like control of traffic, controlling the access of parking lot. License plate recognition involves three components. They deal with an aspect of applying e- government for the improvement in the performance. It is very cost efficient. It has an accurate character recognition of an image that is captured. The disadvantage is that it is suitable to images involving one car at a time eliminating the background [5].

Songke $\mathrm{Li}$ et al, a scientist invented license plate of a vehicle. It is suitable for carriages instead of automobiles. License plates will be registered on their own name, address and phone number. Since the vehicles are becoming more and more, traffic violations increases as well. License plate is a specific data for each automobile and it helps in knowing its owner and contacts him for processing. Recognition of license plate is an important aspect of new generation transportation system because it is simple to identify the license plate number and also the vehicle detection. The major benefit is to develop the exactness of detection and avoids the turbulences. The drawback is that it increases matching time [6].

Al-Ant et al, a scientist invents an innovative traffic system that identifies the vehicle is present or not and behave as such. This system dynamically manages the control of traffic light components based on the alterations of flow of the traffic in various areas. Therefore, increases the efficiency of traffic robs. Also, reduces the time of travel for vehicles. The benefits are limiting pollution, congestion of traffic. The drawback is that timing of alteration of lights even though in there is no traffic [7].

Even though the technology is improved, identifying the theft vehicle is being difficult. Nowadays the thief's are changing the number plate of the theft vehicle and using the theft vehicle. Hence it is difficult to identify the theft vehicle. Already existing systems are finger print recognition and password identification. If the theft vehicle's number plate is replaced by fake number plate, it cannot be identified easily. They have used an edge finding methodology. So we cannot exactly extract the number plate. Hough transform method is used and it requires huge memory and it is expensive. It is applicable only to pages without complex background. Mud and noisy interference leads to imperfect texting [8][9].

Accurate recognition failures due to high speed, dark and rain. In traffic signals, the vehicles have to wait for a long time, since the signals are released sequentially based on timing. Sometimes at one signal there will not be any vehicles but the signal will be opened in that area and we have to wait, there is time for consumption is more. Also each vehicle should carry a RFID tag which is not practically possible. Sensor should be placed in each state of signal which is difficult. More number of sensors should be used, the sensor also does not cover large distance and hence it is difficult to calculate the intensity of vehicles [10].

\section{Proposed System}

In the proposed system, the theft vehicle is identified in toll gates by using license plate recognition method. In the figure I, the license plate is recognized and image is captured by web camera. Once the license plate is recognized, its characters are detected and then matched with the background image. If any mismatch occurs, it 
indicates that the vehicle is theft and it is displayed on the LCD screen in toll gate. Hence the theft vehicle is identified quickly. The recognized license plate also displays the full details of the vehicle's owner on the LCD display. Once when the theft vehicle is identified in the toll gate then the alarm will automatically goes to the ON state where everyone can identify that the vehicle is a theft vehicle. The traffic signal is released based on the high density of vehicles using background subtraction method. By using this method, traffic can be controlled and time consumption is less. An intelligent traffic light system identifies whether the vehicle is present or not. The process flow is shown in figure 1.

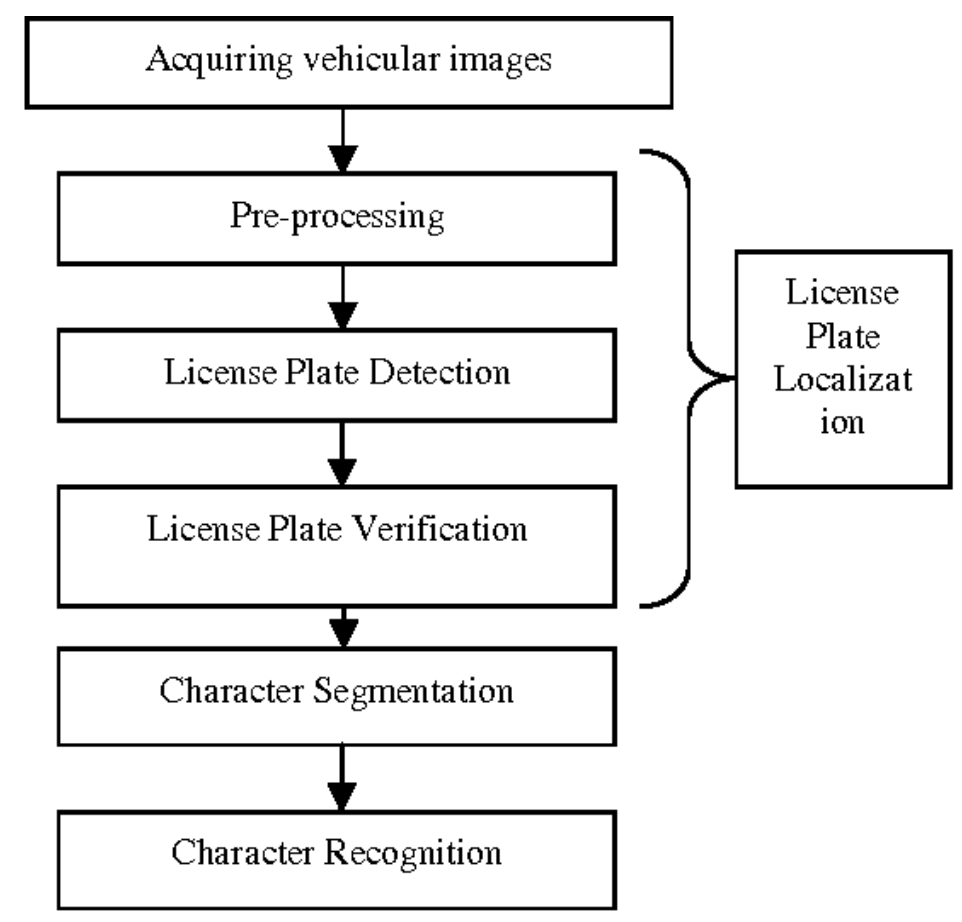

Fig 1: Process flow of the proposed system

Here in figure 2, we are placing one rotating web camera in a traffic signal, where it continuously captures the image and calculates the intensity of the vehicles using background subtraction algorithm. Then it sends the interrupt to the microcontroller that which signal should be released. The microcontroller thus receives the interrupt and releases the corresponding signal. Through this technique we can reduce the traffic light timing by calculating the intensity of vehicles.

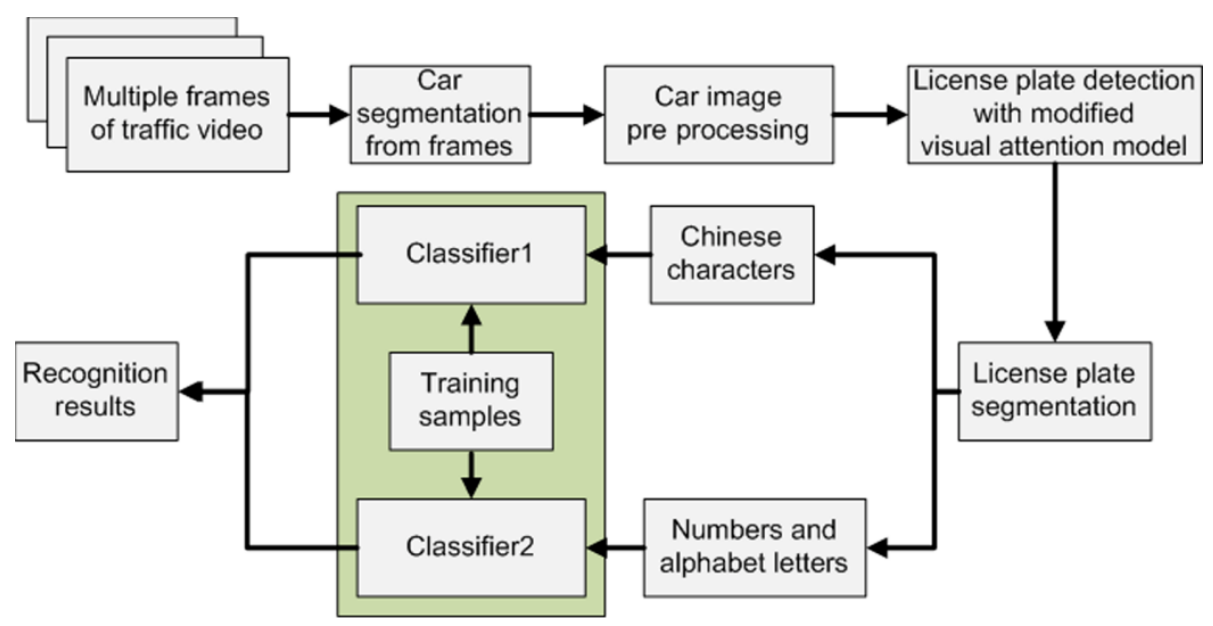

Fig 2: License Plate Detection using Image Processing 


\section{Simulation Results}

The simulations are performed using MATLAB. A device for recording visual image in the form of photograph and transfers input to later processing stage in order to identify the input image. In that they are calculating the image density using proteus tool and give preference to high density of vehicle signal will be open. Hence we can reduce the time for drivers in signal. The license plate detection is used to identify the theft vehicle: the camera in identifying the license plate is employed in extracting the license plate features of an image captured from a car. Usually, values of the pixels in the feature extraction of a license plate are varying because of the components and its background will have a change in the values of the pixel. Now it will be identify the information will be texted in LCD display. It will display ' $A$ ' if it is not theft vehicle and displays ' $B$ ', if it is a theft vehicle. Through this we can identify the theft vehicle. This is shown in figure 3.

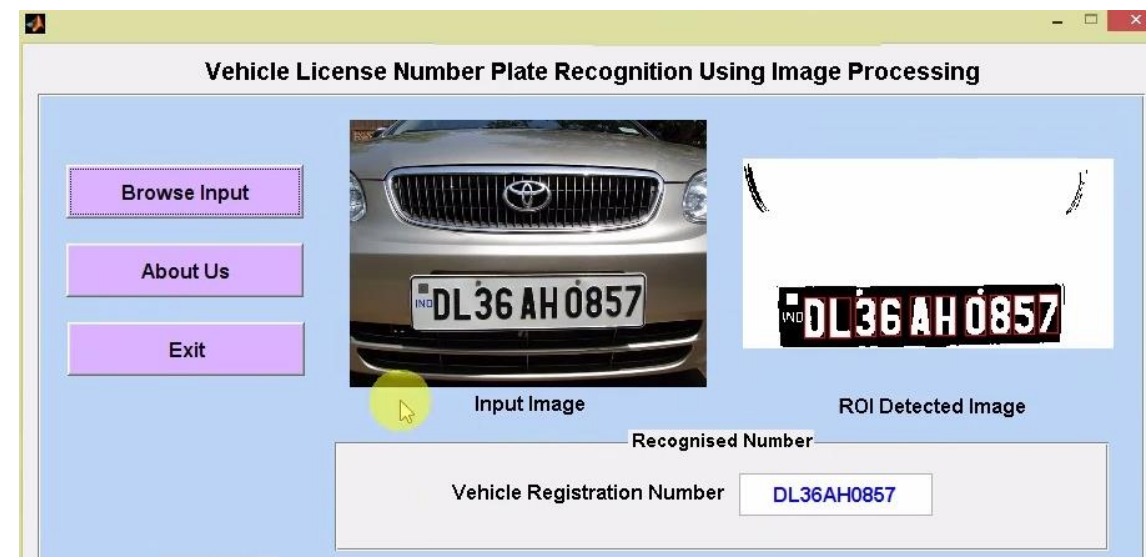

Fig 3: Simulation result of License Plate Detection using Image Processing

ALPR is an important technique in order to detect the number plate of a licensed vehicle by considering an image or a stack of images. The extracted information can be with or without a database in many applications like electronic payment systems and freeway and arterial monitoring devices for traffic surveillance. ALPR employs CC camera, advanced camera or black and white, color camera to capture the image. ALPR is fruitful if the captured images are of good quality. ALPR is a real time application that processes the images of license plates in various conditions like dark or bright times in a day. The histogram results indicating number plate identification is shown in figure 4.

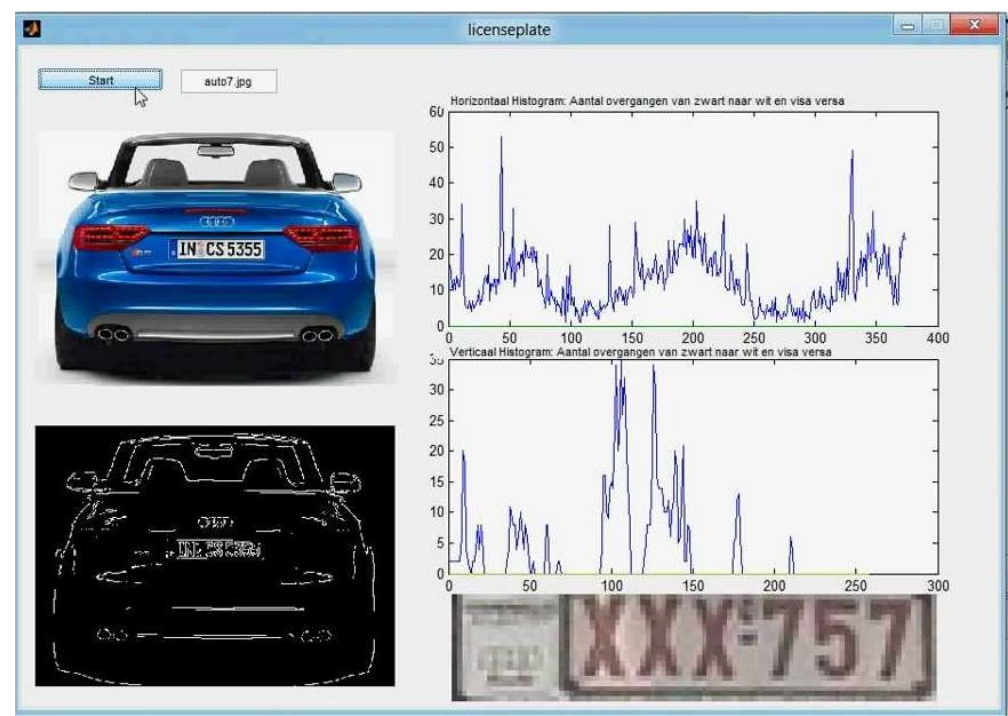

Fig 3: Simulation result showing histogram analysis of License Plate Detection using Image Processing 


\section{Conclusion}

A general technique should be identified to process images in many different countries or states. We should know that the license plate generally consists of various colors, languages, fonts and others have images in the background. Also, these plates are obstructed by mud, light, and some accessories especially on a car. Here, we discuss about methods for ALPR. We classify ALPR based on the features they are used in each method and knowing their advantages, disadvantages, recognition accuracy and processing speed. Managing the timing in traffic controlling by calculating the density of an image. It is done by using object detecting algorithm. The traffic lights can be controlled according by its output. Almost all the traffic light systems functions based on the mechanism of time which alters the lights at regular basis. A traffic light system generally identifies the vehicle present or not and do the need accordingly. The basic key point behind this system is that the vehicle drivers may not spend much time unnecessarily for the lights to alter.

\section{References}

[1] C. E. Anagnostopoulos, I. E. Anagnostopoulos, I. D. Psoroulas, V. Loumos and E. Kayafas, "License Plate Recognition From Still Images and Video Sequences: A Survey," in IEEE Transactions on Intelligent Transportation Systems, vol. 9, no. 3, pp. 377-391, Sept. 2008, doi: 10.1109/TITS.2008.922938.

[2] A. H. Ashtari, M. J. Nordin and M. Fathy, "An Iranian License Plate Recognition System Based on Color Features," in IEEE Transactions on Intelligent Transportation Systems, vol. 15, no. 4, pp. 16901705, Aug. 2014, doi: 10.1109/TITS.2014.2304515.

[3] J. Yang, B. Hu, J. Yu, J. An and G. Xiong, "A license plate recognition system based on machine vision," Proceedings of 2013 IEEE International Conference on Service Operations and Logistics, and Informatics, Dongguan, 2013, pp. 259-263, doi: 10.1109/SOLI.2013.6611421.

[4] H. R. A. Moghassemi, A. Broumandnia and A. R. Moghassemi, "Iranian License Plate Recognition using connected component and clustering techniques," The 7th International Conference on Networked Computing and Advanced Information Management, Gyeongju, 2011, pp. 206-210.

[5] S. H. M. Kasaei and S. M. M. Kasaei, "Extraction and Recognition of the Vehicle License Plate for Passing under Outside Environment," 2011 European Intelligence and Security Informatics Conference, Athens, 2011, pp. 234-237, doi: 10.1109/EISIC.2011.50.

[6] Y. Li, Y. Liu and M. Wang, "Study and Realization for License Plate Recognition System," 2009 AsiaPacific Conference on Information Processing, Shenzhen, 2009, pp. 501-504, doi: 10.1109/APCIP.2009.259.

[7] Mr. Rahul Sharma. (2013). Modified Golomb-Rice Algorithm for Color Image Compression. International Journal of New Practices in Management and Engineering, 2(01), 17 - 21. Retrieved from http://ijnpme.org/index.php/IJNPME/article/view/13

[8] G. Xuezhong, L. Shuang, L. Zhi, L. Yunjiang, S. Taolue and L. Yang, "Research on Automatic License Plate Recognition Technology," 2013 Third International Conference on Instrumentation, Measurement, Computer, Communication and Control, Shenyang, 2013, pp. 750-753, doi: 10.1109/IMCCC.2013.167.

[9] A. E. Rashid, "A fast algorithm for license plate detection," 2013 International Conference on Signal Processing , Image Processing \& Pattern Recognition, Coimbatore, 2013, pp. 44-48, doi: 10.1109/ICSIPR.2013.6497956.

[10] Mr. Kaustubh Patil. (2013). Optimization of Classified Satellite Images using DWT and Fuzzy Logic. International Journal of New Practices in Management and Engineering, 2(02), 08 - 12. Retrieved from http://ijnpme.org/index.php/IJNPME/article/view/15

[11] L. Li and F. Guangli, "The License Plate Recognition System Based on Fuzzy Theory and BP Neural Network," Fourth International Conference on Intelligent Computation Technology and Automation, Shenzhen, Guangdong, 2016, pp. 267-271, doi: 10.1109/ICICTA.2011.77.

[12] Luminoso, L. (2017). Creative engineering. Design Engineering (Canada), 63(1), 30-31. 
[13] Ms. Pooja Sahu. (2015). Automatic Speech Recognition in Mobile Customer Care Service. International Journal of New Practices in Management and Engineering, 4(01), 07 - 11. Retrieved from http://ijnpme.org/index.php/IJNPME/article/view/34

[14] Zhong Qin, Shengli Shi, Jianmin Xu and Hui Fu, "Method of License Plate Location Based on Corner Feature," 2006 6th World Congress on Intelligent Control and Automation, Dalian, 2006, pp. 86458649, doi: 10.1109/WCICA.2006.1713668. 